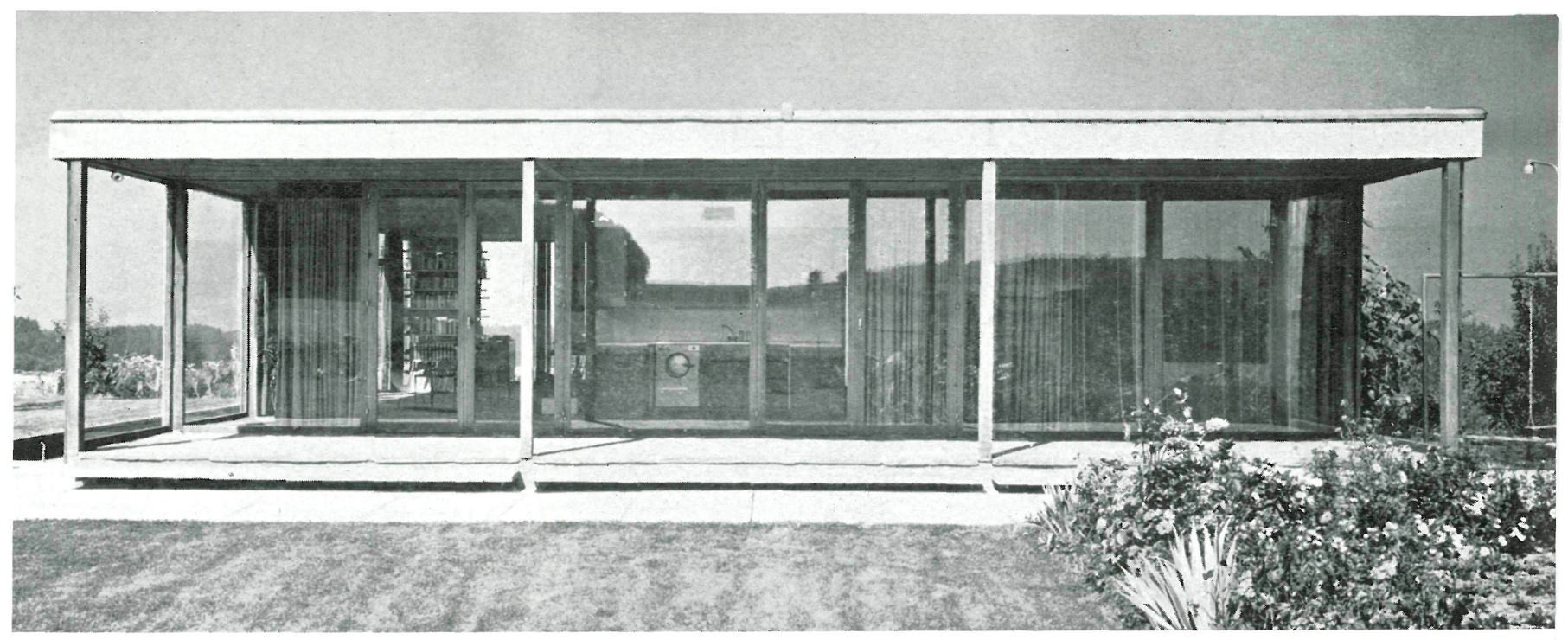

\title{
vivienda unifamiliar Eerca de Solothurn, Suiza
}

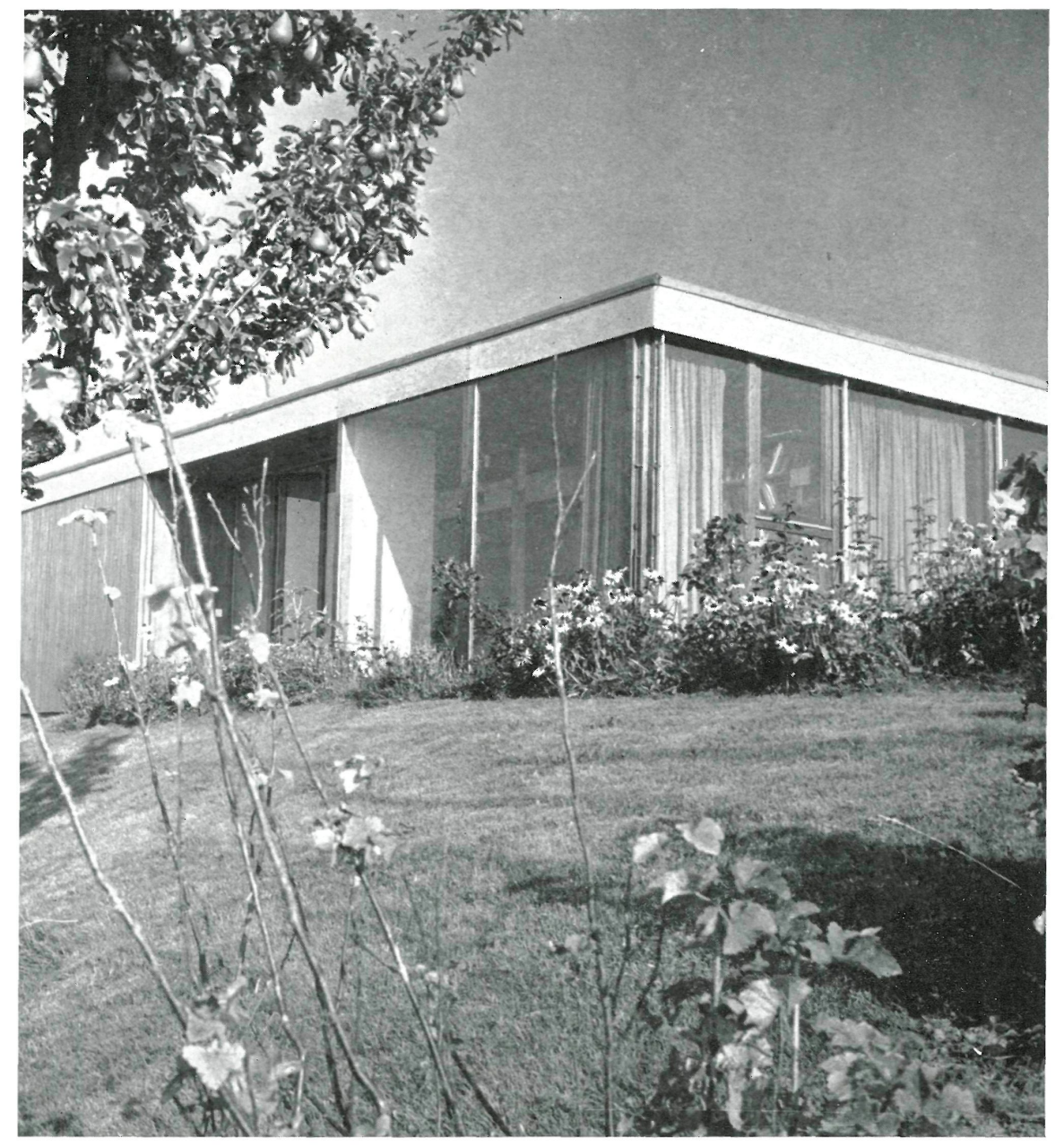

FUEG + HENRY, arquitectos

161.132

\section{sinmopis}

Construida aprovechando el desnivel del terreno, esta vivienda unifamiliar ha sido organizada en dos niveles.

La planta baja alberga: la zona de entrada; estar, dormitorios, y servicio.

Y la de sótano: el garaje, la despensa, un cuarto para «hobby», trastero, y los cuartos para las instalaciones.

La vivienda ha sido funcionalmente modelada en plantas y alzados, atendiendo a sistemas de prefabricación y procurando gran facilidad de movimiento de estancia con paneles móviles. El edificio es gracioso, diáfano y de gran sencillez constructiva y formal. 

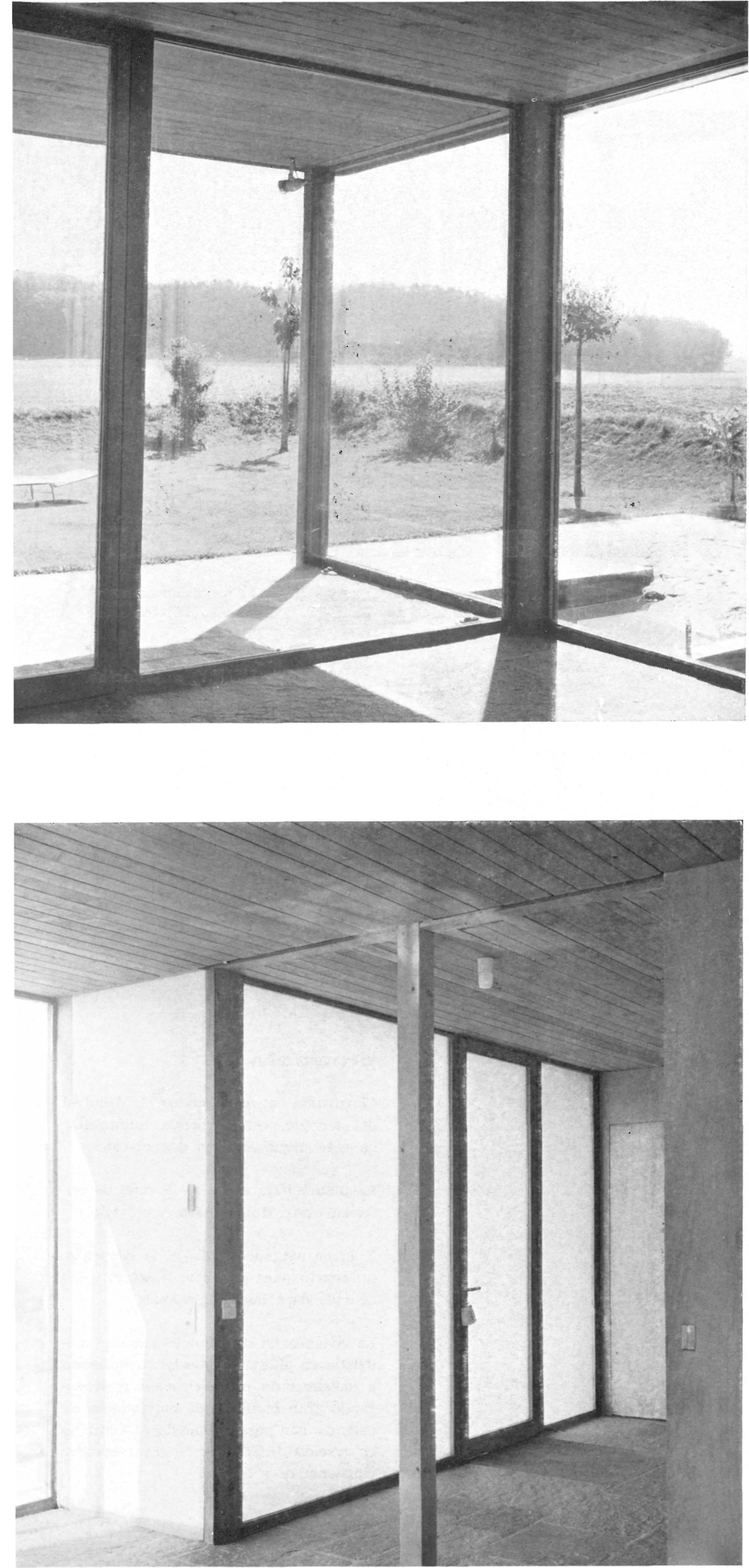

Cerca de Solothurn, en Bucheggberg, Suiza - en medio de un jardín paradisíaco y sobre la falda de una colina, desde la que se domina una bella vista panorámica sobre campos, montes, el Jura y los Alpes-, ha sido construida esta vivienda unifamiliar satisfaciendo el programa de necesidades correspondiente a un matrimonio de maestros con dos niños, de modo que sus moradores puedan atender a sus numerosos invitados.

La construcción ha sido realizada, en parte, por prefabricación y se ha desarrollado en dos niveles.

El nivel correspondiente a la planta noble adopta una forma sensiblemente cuadrada, de $12 \times 12 \mathrm{~m}$, de los que un espacio de $12 \times 2 \mathrm{~m}$ corresponde a la entrada cubierta.

Dicha planta ha sido adecuadamente zonificada, apareciendo claramente diferenciadas: la zona de vivir diurna, la de vivir nocturna, y la de servicios, que ocupa el núcleo central.

Esta zona de servicios, con la cocina y baño, ayuda a incrementar el aislamiento acústico entre las zonas de dormitorios y de estar.

La planta de sótano, además del garaje y la zona de acceso al mismo, contiene: la bodega-despensa, una habitación para «hobby», el trastero, y los cuartos de las instalaciones (acondicionamiento de aire, etc.).

En cuanto a características generales, señalaremos: que las grandes superficies acristaladas en los muros de cerramientos exteriores y las paredes móviles en forma de cortinas en el interior permiten a los usuarios abrir o ce-

Fotos: BERNHARD MOOSBRUGGER 
tramsversial
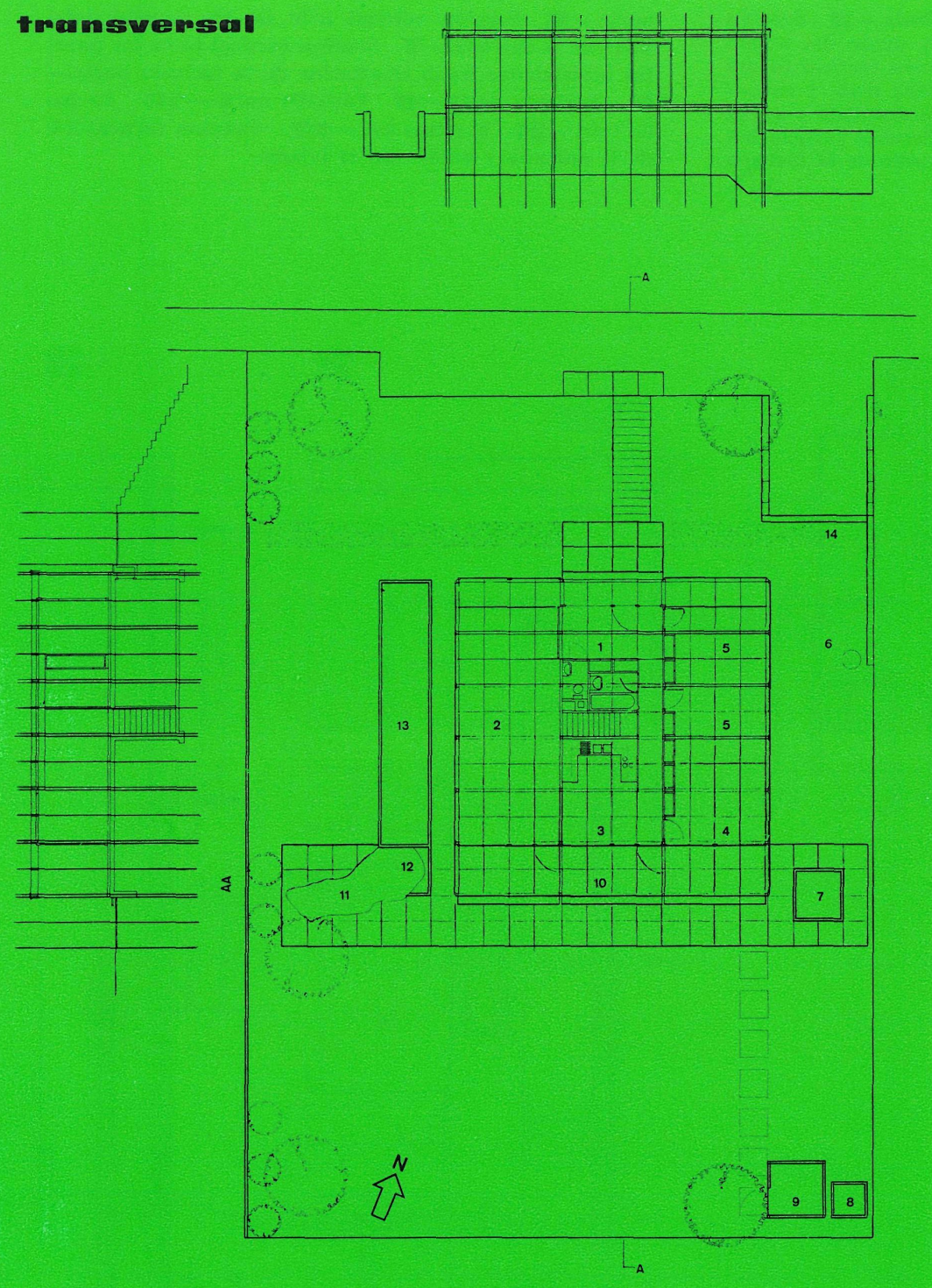

\section{boajo}

1. Entrada, con armarios em. potrados. - 2. Estar. - 3. Co. medor. - 4. Dormitorio padres. ta con pozo -7 Juegos in. fantiles y cajon de aren in. 8. Desecho vegetales. -9 . Uti. es de jardin.-10. Cobertizo. 11. Neolithicum. - 12. Piscina infantil. - 13. Estanque. - 14 . Parterre, con flores.

\section{sótrand}

1. Entrada coches. -2 . Gara je. -3 . Taller. - 4. Trastero.5. Aire acondicionado. -6 . De posito

\section{Secciones y plantas}

1. Sección transversal. -2 . Sección A-A.-3. Planta baja.-4. Sótano. rrar la casa hacia el "exterior», según sus gustos, su estado de ánimo, la temperatura ambiente, la colocación de los muebles, etc.

Debido a dichas grandes superficies acristaladas, la casa se enfría y calienta rápidamente.

En invierno, el intenso soleamiento hace que el consumo de combustible para la calefacción sea menor que el correspondiente a una construcción compacta con ventanas pequeñas.

En el verano, el excesivo calor queda aminorado por medio de las persianas de aluminio y de la ventilación natural, establecida transversal y diagonalmente.

Las exigencias funcionales y emocionales de los usuarios pueden ser satisfechas, de esta forma, de modos muy diversos y a voluntad, en contraste con la edificación tradicional. 
- Sección horizontal: detalle de fachada.- 2 Sección vertical e-e de la pared acristalada y unión del tejado de la fachada este y oeste.- 3 Sección vertical f-f del panel giratorio $y$ del pilar de la fachada oeste $y$ este.- Sección vertical g-g de la pared acristalada y unión de tejado de la fachada norte. -5 Sección vertical h-h de la pared de madera y unión del tejado de la fachada norte.- 6 Sección vertical $h^{\prime} \cdot h^{\prime}$ de las paredes late. rales de la entrada.- $Z$ Sección vertical i-i de las puertas acristaladas hacia el exterior de la fachada norte.8 Fachada oeste.- 9 Fachada norte.-10 Facnada este.-11 Fachada sur.-12 Sección vertical k-k de la entrada y unión con el tejado.
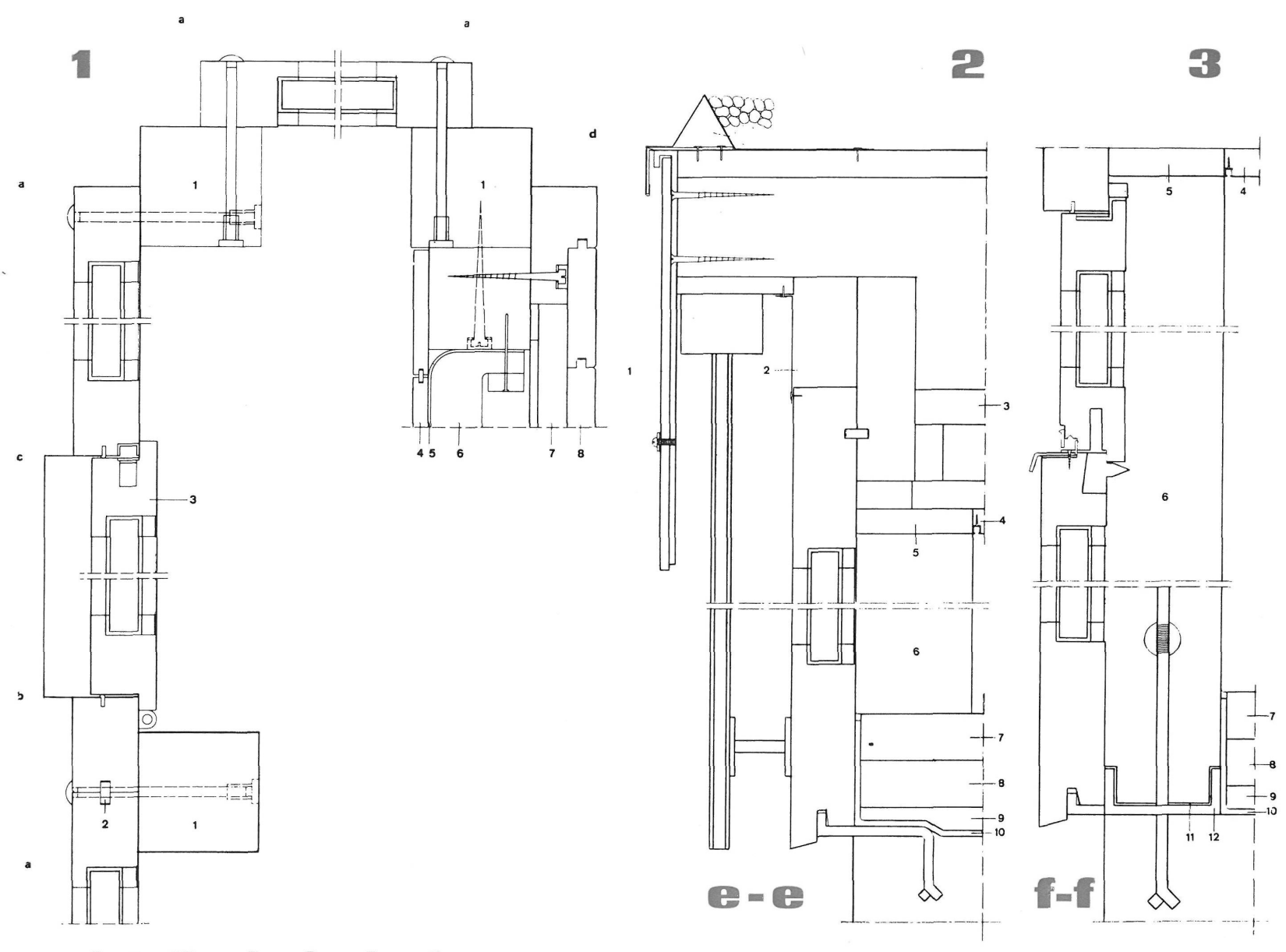

detalle de fachada seceión lnorizontal
1. Pilar de madera de $10 / 10 \mathrm{~cm} .-2$. Muelle de roble. -3 . Panel giratorio.-4. Panel 8. Revestimiento de madera.

Los habitantes de la casa pueden también desmontar las compartimentaciones interiores existentes entre los dormitorios, así como los armarios y los elementos de las puertas; pueden asimismo ordenar los armarios juntos, o unos detrás de otros, ser girados $180^{\circ}$; etc.

Debido a la modulación de la planta, según un módulo de $10 \mathrm{~cm}$ y múltiplos del mismo, cualquier cambio de la distribución o futura ampliación puede ser realizada con relativa facilidad.

Los alzados, sección y detalles han sido asimismo modulados.

La elegante sencillez del conjunto y de cada una de sus partes destaca por su variedad, da la impresión a sus habitantes de estar en libertad, y de que la arquitectura no los enclaustra, sino que se acomoda a sus propios deseos, exigencias y antojos. 
fachadas

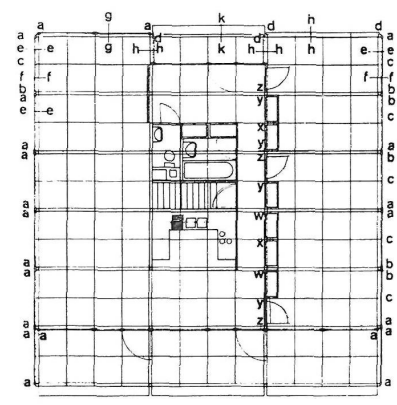

om

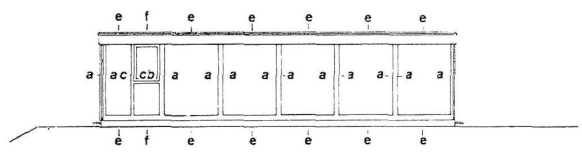

8

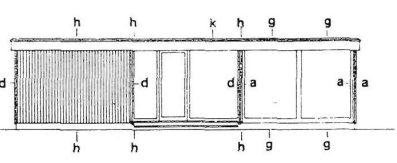

9

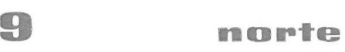

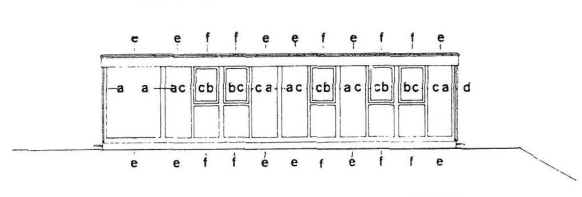

este

10

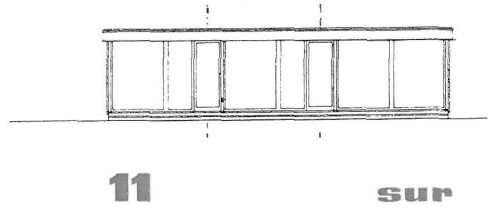

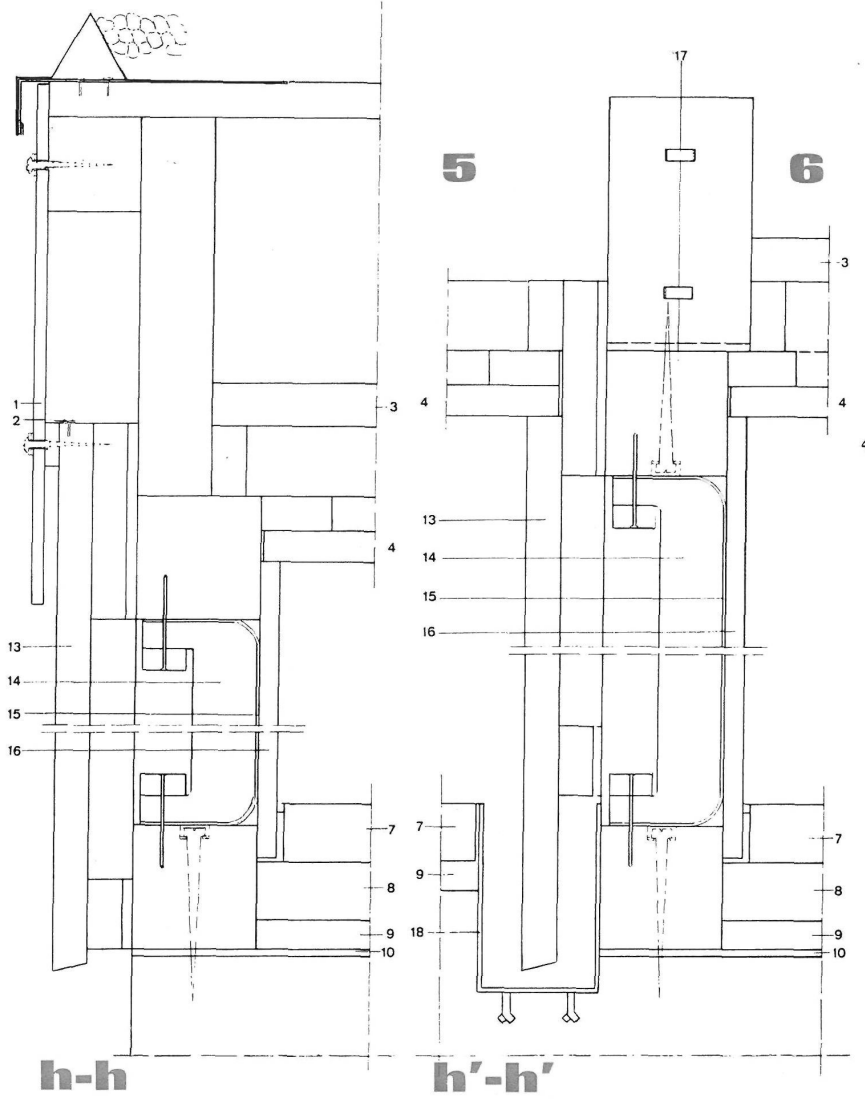

Wi" $=$ in

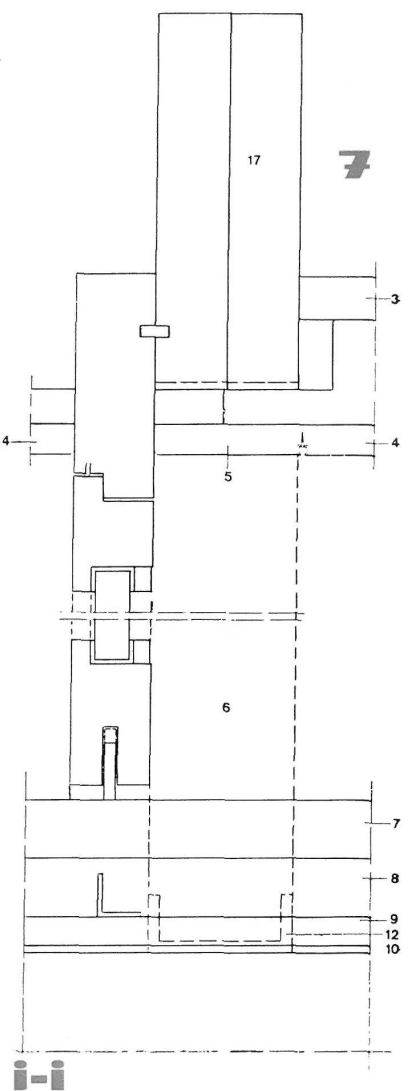

i=i

1. Revestimiento en eternit. -2 . Tela metálica. -3 . Placa de poliestireno.-4. Revestimiento. 5. Tabla entre apoyos. - 6. Pilar de madera de $10 / 10 \mathrm{~cm}$. -7 . Granito de maggia bruto, 11. Lecho de masilla.-12. Anclaje del poliestireno.-10. Aislamiento contra la humedad. miento térmico.-15. Cámara de aire.-16. Panel de aglomerado de madera.-17. Unión entre dos elementos de cubierta.

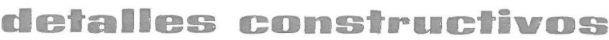

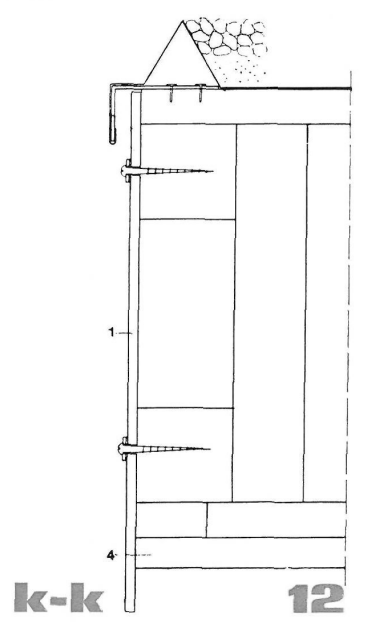




\section{Heabitation unifamiliale prés de Solothumm, Suisse}

Füeg + Henry, architectes

Utilisant le dénivellement du terrain, cette habitation unifamiliale a été construite et organisée en deux niveaux.

Le rez-de-chaussée comprend l'entrée, la salle de séjour, les chambres et les toilettes.

Au sous-sol se trouvent le garage, l'office, une pièce de bricolage, un débarras et les salles destinées aux installations.

Ce logement a été fonctionnellement modelé en plans et façades, en accord avec des systèmes de préfabrication. Une grande facilité de mouvement de séjour est assurée à l'aide de panneaux mobiles. L'immeuble est gracieux, diaphane et de construction très simple et formelle.

\section{Single familg dwelling at Solothurn, Switzeriland}

Füeg and Henry, architects

This house takes advantage of the ground slope, and provides room for a single family on two floor levels.

The ground floor has an entrance zone, the living room, bedrooms, and services.

The basements has a garage, a pantry, hobby and spare rooms, and closets for the installations.

This dwelling has been functionally planned as regards plan and elevation distributions. Prefabrication methods have been adopted and mobile panels have been used to provide ease of adaption to various uses. The building is diaphanous, and of very simple and formal outline.

\section{Einfamiliembaus bei Solothumn - Schweiz}

Füeg + Henry, Architekten

Man hat das Gefälle des Geländes benutzt, um dieses Haus in zwei Niveaus zu bauen.

Das Erdgeschoss beherbergt: die Eingangszone, das Wohnungszimmer, die Schlafzimmer und die Sanitärräume.

Im Untergeschoss liegen: die Garage, die Speisekammer, ein Arbeitszimmer, den Abstellraum und die Installationsräume.

Das Haus wurde mit vorgefertigten Elementen gebaut und im Innen hat man bewegliche Platten benutzt. Das Gebäude ist von grosser aufbauenden Einfachheit. 